\title{
Dexamethasone and flavor neophobia in hamsters
}

\author{
RODERICK WONG, CHRISTOPHER B. MCBRIDE, and MARNE OWEN \\ University of British Columbia, Vancouver, British Columbia, Canada
}

\begin{abstract}
The effects of dexamethasone (DEX) on the reactions of male and female hamsters to novel flavors were studied in an attempt to assess the role of novelty-induced stress in flavor neophobia. DEX is a synthetic glucocorticoid of wide-ranging effects including the blocking of adrenal response to stress in pregnant hamsters. If reactions to novel flavors are mediated by stress, DEX-treated hamsters should show an attenuation of flavor neophobia in relation to nontreated animals. Neophobic reactions were assessed by comparing the hamsters' latency to pouch a novel-flavored nut in comparison with a familiar unflavored one. Flavor neophobia was manifested in response to novel salty, sweet, or sour nuts when male and female hamsters were tested under the control (no-DEX) condition. Similar results were obtained with DEX-treated animals, with one exception. There was an interaction indicating that DEX-treated females showed enhanced neophobic reactions to a sucroseflavored nut. The theoretical implications of these findings are discussed.
\end{abstract}

In a recent series of experiments, Wong and McBride (1993) have demonstrated that male gerbils and hamsters exhibit a neophobic reaction when they encounter sweet, salty, or sour nuts for the first time. This was expressed by an aversion toward the flavored nut, as indicated by a longer latency to either ingest (gerbils) or pouch (hamsters) it as opposed to an unflavored one. This initial aversion declined over subsequent presentations as the flavor became more familiar. The latency measure used in assessing neophobia in these experiments is more sensitive than the traditional one involving measures of the animals' intake. Because neophobia is a short-lived phenomenon, Carroll, Dinc, Levy, and Smith (1975) argued and demonstrated that it is best detected in a brief test.

Novel foods are typically approached with hesitation, in a manner that suggests possible conflicting tendencies (Rozin, 1977). Such reactions may reflect general effects elicited by novel stimuli (Corey, 1978; Halliday, 1966; Russell, 1973). Corey regarded neophobia as a specific instance of neotic behavior, a term that refers to a range of responses to novel stimuli, including exploration, aggression, orientation, and the like. Novelty is a function of the discrepancy between past experience and present sensation; the greater the discrepancy, the greater the novelty. More specifically, Domjan (1977) interpreted the rodents' avoidance of novel or unfamiliar flavors as a reaction to the assumed aversive properties of such novel stimuli. From this perspective, preexposure of the

This research was supported by a research grant from NSERC of Canada to R. W. We thank P. Best, J. Misanin, and a third (anonymous) reviewer for their careful reading and detailed critical comments and suggestions, which led to the present (improved) version of this paper. Requests for reprints should be sent to R. Wong, Department of Psychology, University of British Columbia, Vancouver, BC, Canada V6T 1 Z4. rodent to the test stimuli reduces their relative novelty and hence the animal finds them less aversive. Thus, neophobia in response to the test stimuli would be attenuated as a result of such experience. This interpretation accounts for the finding that the degree of neophobia decreases as the number of exposures to the novel food increases (Nachmann, Rauschenberger, \& Ashe, 1977).

In the present experiments, we studied the effects of a synthetic glucocorticoid, dexamethasone (DEX), on flavor neophobia in hamsters. There are various reasons why this steroid with wide-ranging effects may influence animals' reactions to novel stimuli. This substance binds to corticosterone receptors in the pituitary gland and prevents the release of adrenocorticotrophic hormone (ACTH) and $\beta$-endorphin (Guillemin et al., 1977). Data on the suppressive effects of DEX on ACTH liberation in rats are considerable (Ahlers \& Richardson, 1985; Beatty, Beatty, Bowman, \& Gilchrist, 1970; Heinsbroek, van Oyen, van De Poll, \& Boer, 1983; Henessey, Smotherman, \& Levine, 1980; Levine \& Levin, 1970). DEX has also been effective in supressing adrenal reaction to stress in pregnant hamsters (Pratt \& Lisk, 1990).

Aside from its effects on the stress system, DEX affects the metabolic processes. Grigson, Johnson, Collier, and Flaherty (1989) found that DEX caused a decrease in body weight in rats and suggested that this was partially due to the decrease in food intake. In contrast, Lowy and Yim (1982) observed that although DEX did not cause a reduction of food intake in nonstressed hamsters, it decreased the body weight of these rodents (Lowy \& Yim, 1982). The latter attributed the weight loss to direct catabolic effects of this glucocorticoid.

Recent work on "emergence neophobia" reported in this journal indicates that in rats, choice between a novel and a familiar alley may be mediated by an interaction 
of glucocorticoid hormones and habituation (Maren, Patel, Thompson, \& Mitchell, 1993). These experimenters demonstrated that emergence behavior is highly correlated with basal plasma corticosterone levels measured 2 weeks after the animals had been tested in the emergence apparatus. Interestingly enough, neophobic rats had relatively lower plasma corticosterone levels than did neophilic rats. Although the tasks are different, flavor neophobia and emergence neophobia are forms of neotic behavior which may be mediated by similar processes. Results obtained by Mitchell, Fairbanks, and Laycock (1977); Mitchell, Kirschbaum, and Perry (1975); and Mitchell, Koleszar, and Scopatz (1984) indicate that the interaction of habituation and arousal is involved in emergence neophobia. Because of the pervasive physiological effects of DEX, this corticosterone agonist was expected to influence changes in reactions to novel flavors. On the basis of Maren et al.'s (1993) findings, one would predict that DEX should enhance flavor neophobia, but from the perspective of the novelty-stress hypothesis, DEX should attentuate flavor neophobia.

\section{GENERAL METHOD}

\begin{abstract}
Subjects
The subjects were 38 male and 32 female Syrian golden hamsters (Mesocricetus auratus) that were 6-18 months old at the beginning of the experiment. The body weight of the males ranged from 79 to $145 \mathrm{~g}$ and that of females from 75 to $135 \mathrm{~g}$. The animals were individually housed in $15 \times 19 \times 27 \mathrm{~cm}$ plastic cages with Sanicel bedding and pieces of shredded paper for nesting placed on the bedding. The hamsters were housed in a colony maintained at $24^{\circ} \mathrm{C}$ on a $12: 12$-h light:dark cycle, with light onset at $0800 \mathrm{~h}$ and offset at $2000 \mathrm{~h}$.

The animals were maintained on Purina chow and water or DEX solution ad lib during the duration of each experiment. Prior to the experiments, these animals had never been exposed to any nourishment other than Purina chow and tap water. The body weights of the animals were measured periodically during different stages of the experiment in order to obtain an independent assessment of the effects of DEX. This substance has been shown to reduce hamsters' body weight (Lowy \& Yim, 1982).
\end{abstract}

\section{Materials}

DEX (Sigma, No. D-1756) was dissolved into distilled water at a concentration of $1.4 \mu \mathrm{g} / \mathrm{ml}$. Because some hamsters drank less than $10 \mathrm{ml}$ of fluid/day, $1.4 \mu \mathrm{g} / \mathrm{ml}$ was used to ensure that all hamsters received a minimum DEX dose of $10 \mu \mathrm{g} /$ day, a dose shown to reduce stress in hamsters (Pratt \& Lisk, 1990). Fresh DEX solution was prepared every 3 days, and the solution sat for at least $24 \mathrm{~h}$ prior to use. The control animals received distilled water as their drinking fluid.

The food item used in testing, a blanched peanut, was flavored by being rolled in $\mathrm{NaCl}$, sucrose, or citric acid. The crystals of these three compounds were ground to a fine consistency so that the peanut was evenly coated with the compound.

\section{Procedure}

Blind behavioral testing (with respect to the treatment conditions) was done in the home cage, and the animals were not food deprived during the tests. Each animal had access to $30 \mathrm{ml}$ of drinking solution each day. A plain or flavored peanut was dropped into the corner of the cage opposite the nesting area, and an elec- tronic timer was turned on when the nut was first sniffed and turned off when the hamster pouched the nut (oral latency). In Experiments 1 and 2 , involving tests with salted and sweetened nuts, the hamsters were given six daily test trials; and in Experiment 3, involving sour-tasting nuts, the animals, were given eight daily test trials.

\section{EXPERIMENT 1}

It has been previously established that hamsters exhibit neophobic reactions to a peanut coated with $\mathrm{NaCl}$ (Wong \& McBride, 1993). If neophobia in response to the salt flavor is mediated by an interaction of glucocorticoid hormones and habituation, the oral latencies of DEX-treated hamsters to the salt-flavored nut should differ from those of control animals, which drank distilled water.

\section{Method}

To ensure that neophobic reactions in the experiments were due to novel flavors rather than novel foods, all the hamsters were exposed to unflavored peanuts for 10 daily sessions, at which time they showed stable asymptotic oral latency. Male and female hamsters were randomly assigned (with some counterbalancing for age and body weight) to the four groups designated as G1, G2, G3, and G4. A single plain peanut was dropped in the cage, and the oral latency was recorded. Three days after these baseline trials, the animals were tested for flavor neophobia.

Three days before the test trials, the water for Groups G1 and G2 was replaced with DEX solution, while Groups G3 and G4 continued to receive distilled water. The water consumption was measured daily, and the containers were refilled with their respective solutions as described in the General Method section. During this 3-day preexperimental phase, no nuts were given to any of the animals. Then Groups $\mathrm{G} 1$ and $\mathrm{G} 3$ were tested with salted nuts, and Groups G2 and G4 were tested with plain nuts. The oral latency of these animals was measured during six daily trials.

\section{Results and Discussion}

During the baseline trials, there were no significant differences among the groups or between sexes $(p>$ $.05)$, but there was a significant trials effect $[F(9,261)=$ $12,20, p<.001]$. This effect indicated that there was an initial neophobic response (long latency) to the peanut $(M=224.43 \mathrm{sec}$ on Day 1), which decreased over the subsequent test trials $(M=4.69 \mathrm{sec}$ on Day 10$)$.

An analysis of variance (ANOVA) of the animals' response latencies during the experimental phase indicated significant differences arising from flavor $[F(1,62)=$ $34.69, p<.001]$, but no significant differences due to either DEX $(p>.05)$ or sex $(p>.05)$. There were clearly changes over test trials $[F(5,310)=25.27, p<.001]$, as well as one arising from the interaction of flavor by trials $[F(5,310)=19.81, p<.001]$. An analysis of simple main effects revealed that although the groups showed different levels of responding during the initial test trial, they did not differ on the subsequent test trials $(p>.05)$. More specifically, this interaction was due to the longer latencies of the hamsters tested with the novel, salted nut during Day 1 relative to those tested with the familiar, unflavored nut. 
A graphic depiction of these results is shown in Figure 1. A multiple comparison of the groups with the Newman-Keuls test on Day 1 revealed significant differences $(p<.01)$ between the groups tested with the salted nut and the plain nut. It is evident that neophobia in response to the salted nut was evident in both males and females tested under the DEX and no-DEX conditions.

Although we had predicted that DEX would decrease the hamsters' oral latency of salted nuts when compared with those who did not receive this treatment, the results did not support this prediction. Similarly, the DEX treatment did not affect the response latencies of male and female hamsters tested on the plain nut in comparison with control nontreated animals.

In general, the results of this experiment demonstrated the manifestation of flavor neophobia and indicated that it was not attenuated through treatment with DEX. Such results pose difficulties for the hypothesis that flavor neophobia may be mediated through stress experience following exposure to a novel flavor.

\section{EXPERIMENT 2}

Sweet flavors are associated with high caloric intake, and, like salty flavors, they elicit a positive hedonic reaction in rats (Grill \& Berridge, 1985). Hamsters show greater preference for sucrose solution than for unflavored water (Carpenter, 1956). Although intake of sweetened fluids and foods may be greater than that of plain substances when the animals are tested over a period of time, we predict that, as with a salty-flavored nut, a sweetened one should elicit a neophobic reaction when hamsters first encounter it, and that this reaction would eventually dissipate. Although we had predicted that DEX would influence the animals' oral latency to the salt-coated nut, the results of Experiment 1 did not support this hypothesis. The present experiment was conducted to investigate such a possibility in the hamsters' reactions to a novel sugar-coated nut.

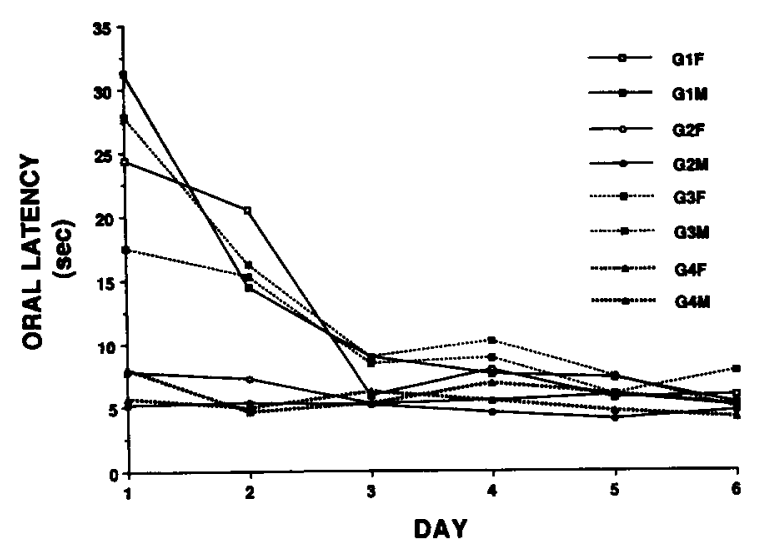

Figure 1. Latency of male and female hamsters to pouch an $\mathrm{NaCl}-$ flavored (Gps G1 and G3) or unflavored (Gps G2 and G4) nut, resulting from daily ingestion of dexamethasone (Gps G1 and G2) or water (Gps G3 and G4), over six trials.

\section{Method}

The same hamsters and groupings from the previous experiment were used; Groups G1 and G2 continued to receive DEX treatments, and Groups $\mathrm{G} 3$ and $\mathrm{G} 4$ received distilled drinking water. The two groups receiving the unflavored peanuts in the previous experiments, G2 and G4, were now tested with sugar-coated peanuts in this experiment. The remaining two groups, G1 and G3, received unflavored peanuts. Behavioral testing was performed in the manner described in the General Method section. These six daily trials were given after the animals received 2 days of assessment with a plain peanut following their respective experiences in Experiment 1 .

\section{Results and Discussion}

An ANOVA revealed flavor effects $[F(1,62)=23.58$, $p<.001]$, but no DEX or sex differences $(p>.05)$. There were also effects arising from trials $[F(5,310)=19.76$, $p<.001]$ and their interaction with flavor $[F(5,310)=$ $18.05, p<.001]$. As in Experiment 1, these results indicate flavor differences during the 1st test day, and these differences disappeared as the groups tested with the sugar-coated nut became habituated to the novel flavor (Figure 2). There was also a significant interaction involving flavor, $\mathrm{DEX}$, sex, and trials $[F(15,310)=3.08$, $p<.001]$.

Multiple comparisons with the Newman-Keuls test on Day 1 indicated that both male and female hamsters tested under the no-DEX condition exhibited neophobic reactions to the sweetened nut $(p<.01)$. Under the DEX condition, an inspection of Figure 2 hinted that DEXtreated male hamsters took longer to pouch the sweetened nut $(M=24.2 \mathrm{sec})$ as opposed to the plain nut $(M=$ $5.6 \mathrm{sec})$. However, the difference was not statistically significant $(p>.05)$, because of the high intragroup variability of the subjects. In contrast, the extremely long latency of the DEX-treated females' reactions to a sucroseflavored nut $(M=82.9 \mathrm{sec})$, which was greater $(p<.01)$ than those in the control condition $(M=39.67 \mathrm{sec})$ was surprising and unexpected. The neophobia to this flavor that was manifested under control conditions was enhanced in females as a result of DEX.

\section{EXPERIMENT 3}

In this experiment, we studied the effects of citricacid-flavored nuts on the hamsters' latency to pouch such nuts relative to unflavored ones. Because of the negative results of Experiment 1 and the interaction in Experiment 2 indicating enhanced neophobia among DEX-treated females to a sucrose-flavored nut, it is critical to determine whether this agent would influence neophobic reactions to a novel flavor that was hedonically negative.

\section{Method}

A new set of naive hamsters was used in this experiment. The subjects were 38 male and 32 female Syrian golden hamsters whose ranges in age and body weight were similar to those in the previous experiments. They were housed and tested under similar conditions, with the exception that the experimental groups, G1 and G3, were tested with plain nuts under DEX or no-DEX con- 


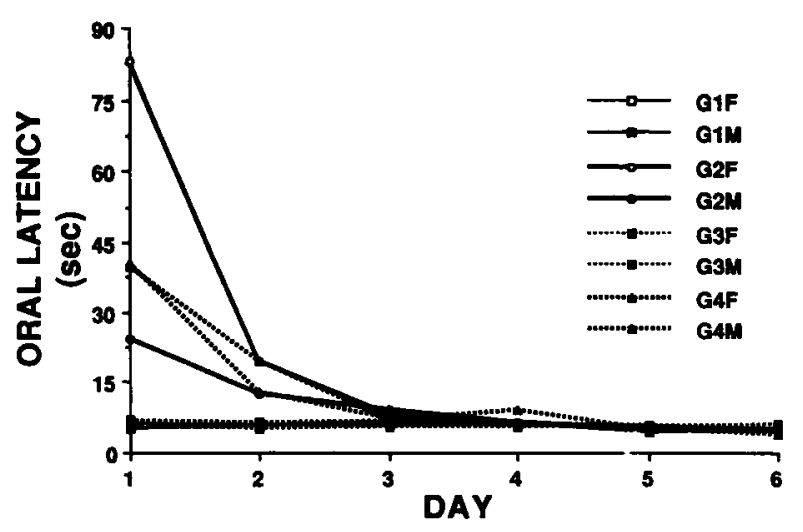

Figure 2. Latency of male and female hamsters to pouch a sucrose-flavored (Gps G2 and G4) or an unflavored (Gps G1 and G3) nut, resulting from daily ingestion of dexamethesone (Gps G3 and G4) or water (Gps G1 and G2), over six trials.

ditions, respectively. Groups G2 and G4 were tested with citric-acid-flavored nuts under DEX and no-DEX conditions, respectively. The oral latency of these animals' responses were measured during eight daily trials.

\section{Results and Discussion}

An ANOVA indicated that there were flavor differences $[F(1,62)=48.51, p<.001]$ but none due to either DEX or sex $(p>.05)$. As with the other experiments, there were changes arising from trials $[F(7,430)=$ $15.50, p<.001]$, and there was a significant flavor $\times$ trials interaction $[F(7,430)=15.71, p<.001]$. The latter was due to the longer latencies of the hamsters tested with the novel sour nut during the first test trial as opposed to those tested with the familiar unflavored nut. These results are displayed in Figure 3.

The Newman-Keuls test on group differences on Day 1 indicated that the groups tested with the citricacid-flavored nut took longer to pouch it than did those tested with a plain nut. Both DEX and non-DEX animals showed a longer latency to pouch the citric-acid-flavored nut as opposed to an unflavored one. Multiple comparisons of the groups on Day 2 did not reveal any significant difference among them $(p>.05)$. Such results indicate that although hamsters exhibited neophobic reactions to the sour flavor, the DEX treatment did not influence the animals' reactions to either flavored or unflavored nuts.

\section{GENERAL DISCUSSION}

In general, these experiments indicated that flavor neophobia in hamsters was not attenuated by the synthetic glucocorticoid dexamethasone (DEX) as originally predicted. Given the pervasive physiological effects of DEX, our failure to observe an effect is worthy of note. Because of the effectiveness of DEX in suppressing adrenal reactions to stress in hamsters (Pratt \& Lisk, 1990), we believe that the animals in our experiments experienced similar effects. In the present exper- iments we were certain that the hamsters ingested at least $10 \mu \mathrm{g}$ of DEX per day, an amount that Pratt and Lisk found effective in reducing stress in pregnant hamsters. Although we do not have data to verify the effectiveness of DEX in blocking the novelty-induced corticosterone response, we obtained evidence indicating body weight loss of the DEX-treated groups relative to those given distilled water. Although the groups did not differ in body weight during the beginning of the experiments $(M=110.03$ and $110.21 \mathrm{~g}$ for those assigned to the DEX and no-DEX groups, respectively), at the end of the experiments, those given DEX showed a decrease $(p<.05)$ in body weight $(M=102.22)$ compared with the no-DEX animals $(M=114.3 \mathrm{~g})$.

Previous experiments have clearly demonstrated the effects of DEX on body weight loss in rats (Devenport \& Stith, 1992; Grigson et al., 1989) and hamsters (Lowy \& Yim, 1982). Devenport and Stith state that DEX is a Type II corticosteroid agonist which has negligible effects on total food intake and retards normal weight gain. Thus, the function of DEX (corticosterone Type II action) is primarily metabolic and explains Lowy and Yim's findings of DEX effects of body weight reduction without a corresponding decrease in food consumption in hamsters. The fact that we obtained a similar effect in our animals indicates that this DEX-treatment had a physiological effect.

It is possible that subcutaneous injections of DEX may be more effective than its introduction in the drinking water of the experimental animals. However, the former method would result in stress owing to handling and injection, and the simple noninvasive method avoids the elevation of cortisol independent of the possible novelty stress elicited during testing.

Although DEX did not have a general effect on neophobia to most flavors, the results in Experiment 2 revealed an intriguing sex $\times$ treatment interaction. There is an indication that the female hamsters' reactions to a

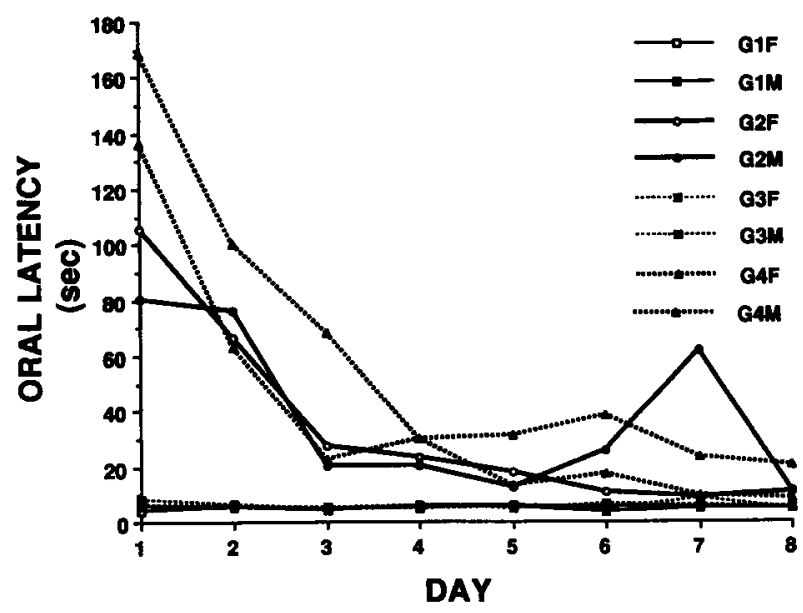

Figure 3. Latency of male and female hamsters to pouch a citricacid-flavored (Gps G2 and G4) or an unflavored (Gps G1 and G3) nut, resulting from daily ingestion of dexamethasone (Gps 1 and G2) or water (Gps G3 and G4), during eight trials. 
novel sucrose-flavored nut are different from their reactions to other novel flavors. Although males showed neophobia to such a stimulus under the no-DEX condition, they failed to react similarly when given DEX. In contrast, the DEX-treated females showed greater neophobia in response to this flavor than did those tested under the no-DEX condition. Such results are the opposite of predictions from the novelty-stress hypothesis but are congruent with Maren et al.'s (1993) finding of lower plasma corticosterone levels among rats that showed high emergence neophobia. Although the control animals' reactions to a novel sweetened nut are consistent with their reactions to other flavored nuts, DEX seems to affect hamsters differently when they are tested with sucrose-flavored nuts.

In his analysis of the "eating paradox," Woods (1991) argued that foods with powerful positive reinforcing qualities also pose a potential threat to organisms. The act of eating is a disruptive event in a homeostatic sense, given that unfamiliar, calorie-rich foods may challenge the animal's cephalic (anticipatory) insulin response. The initial hypophagia shown by animals placed on a novel rich diet may be seen as an adaptive avoidance of such "glucose excesses." As a consequence of repeated experiences, the animal is able to consume more of the diet because of adjustments in the anticipatory reactions that regulate the release of insulin. Thus, a neophobic reaction to a novel (and potentially rich or toxic) food is adaptive. So, why would DEX enhance female hamsters' neophobic reactions to sweet-tasting foods?

Blass (1987) has suggested that opioid release confers the positive affective state elicited by sweet taste. He cited LeMagnen, Marfaing-Jallat, Miceli, and Devos's (1980) experiments that indicated a decrease in preference for sweet solutions among rats administered an opioid antagonist. Blass predicted that smaller volumes of sugar solutions would be ingested to the extent that less opioid was effectively available under conditions of receptor blockade. Similarly, Cooper, Jackson, Kirham, and Turkish (1988) proposed that opiate antagonists attenuate the palatability of food associated with oropharyngeal stimulation. From this perspective, the administration of DEX which prevents endorphin release via its inhibition of ACTH liberation not only decreases sucrose intake but should increase the hamsters' response latency to sweet-tasting foods, especially novel ones. However, the latter effect was evident only among female hamsters given DEX.

Although there are no published data on sex differences in sucrose preference among hamsters, Wong (1985) found that the saccharin preference of females was greater than that of males in a "two-food" test when this sweetener was added to a liquid diet, Isocal. Sex differences were evident when the concentrations of saccharin were $0.1 \%$ and $0.3 \%$, but not at higher concentrations. The preference ratios (saccharin diet/ saccharin diet + unflavored diet) for females were 0.68 and 0.62 for the $0.1 \%$ and $0.3 \%$ concentrations, respectively, while the correponding ratios for males were 0.45 and
0.49. It appears that female hamsters are more responsive to sweet-tasting liquid diets than males. If palatability is decreased as a result of opioid antagonists, then DEX effects would be more evident in females than males.

Data from hamsters tested under the no-DEX conditions in the present experiment replicated those tested under comparable conditions in Wong and McBride's (1993) study and showed that nontreated females behaved similarly to nontreated males. Flavor neophobia in response to salty, sweet, and sour nuts was clearly demonstrated, and the oral latencies of the hamsters in the respective conditions in these experiments were very similar. It would appear that the present method is a reliable behavioral assay for assessing flavor neophobia and agents that influence the processes.

Recent research has indicated that exposure to novel environmental stimuli alters the animals' pain threshold in that they are hypoalgesic in comparison with animals previously familiarized with these stimuli (Abbott, Franklin, \& Connell, 1986; Bardo \& Hughes, 1979; Gamble \& Milne, 1989; Rochford \& Stewart, 1987; Sherman, 1979). Such novelty-induced hypoalgesia was found to be resistant to pretreatment with naloxone (Rochford \& Stewart, 1987), but it was reversed with the pretreatment of the noradrenergic antagonist, clonidine (Rochford, 1992). Perhaps flavor neophobia is mediated by nonopioid, rather than opioid, substrates affected by the glucocorticoid, DEX. If novelty-induced hypoalgesia and flavor neophobia are mediated by similar mechanisms, selective noradrenergic agonists and antagonists may have differential effects on the hamsters' oral latency to a nut coated with a novel flavor. Alternatively, stress may have little to do with flavor neophobia.

\section{REFERENCES}

AbbotT, F. V., Franklin, K. B., \& Connell, B. (1986). The stress of a novel environment reduces formalin pain: Possible role of serotonin. European Journal of Pharmacology, 126, 141-144.

Ahlers, S. T., \& Richardson, R. (1985). Administration of dexamethasone prior to training blocks ACTH-induced recovery of an extinguished avoidance response. Behavioral Neuroscience, 99, 760-764.

BARDo, M. T., \& Hughes, R. A. (1979). Exposure to a nonfunctional hot plate as a factor in the assessment of morphine-induced analgesia and analgesic tolerance. Pharmacology, Biochemistry \& Behavior, 10, 481-485.

Beatty, P. A., Beatty, W. W., Bowman, R. E., \& Gilchrist, J. C. (1970). The effects of ACTH, adrenalectomy and dexamethasone on the acquisition of an avoidance response in rats. Physiology \& Behavior, 5, 939-944.

BLASs, E. M. (1987). Opioids, sweets and a mechanism for positive affect: Broad motivational implications. In J. Dobbing (Ed.), Sweetness (pp. 113-123). London: Springer-Verlag.

CARPENTER, J. A. (1956). Species differences in taste preference. Journal of Comparative \& Physiological Psychology, 49, 139-144.

Carroll, M. E., Dinc, H. I., Levy, C. J., \& Smith, S. C. (1975). Determinants of neophobia and enhanced neophobia in the albino rat. Journal of Comparative \& Physiological Psychology, 89, 457467.

CoOper, S. J., JaCKSON, A., Kirkham, T. C., \& TURKish, S. (1988). Endorphins, opiates and food intake. In R. J. Rodgers \& S. J. Cooper (Eds.), Endorphins, opiates and behavioural processes (pp. $141-$ 186). London: Wiley. 
COREY, D. T. (1978). The determinants of exploration and neophobia. Neuroscience \& Biobehavioral Reviews, 2, 235-253.

DEVENPORT, L., \& STITH, R. (1992). Mimicking corticosterone's daily rhythm with specific receptor agonists: Effects on food, water, and sodium intake. Physiology \& Behavior, 51, 1247-1255.

Domuan, M. (1977). Attenuation and enhancement of neophobia for edible substances. In L. M. Barker \& M. Domjan (Eds.), Learning mechanisms in food selection (pp. 151-179). Waco, TX: Baylor University Press.

Gamble, G. D., \& Milne, R. J. (1989). Repeated exposure to sham testing procedures reduces reflex withdrawal and hot-plate latencies: Attenuation of tonic descending inhibition? Neuroscience Letters, 96, 312-317.

Grigson, P. S., Johnson, D. F., Collier, G., \& Flaherty, C. F. (1989). The effects of dexamethasone-21-acetate on meal size, meal frequency and macronutrient self-selection in rats. Physiology \& Behavior, 46, 211-216.

GrILl, H. J., \& BERRIDGE, K. C. (1985). Taste reactivity as a measure of the neural control of palatability. Progress in Psychobiology \& Physiological Psychology, 11, 1-61.

Guillemin, R., Vargo, T., Rossier, J., Minick, S., Ling, N., RivIER, C., VALE, W., \& BLOOM, F. (1977). Beta-endorphin and adrenocorticotropin are secreted concomitantly by the pituitary gland. Science, 197, 1367-1369.

HALliday, M. S. (1966). Exploration and fear in the rat. Symposia of the Zoological Society of London, 18, 45-59.

Heinsbroek, R. P. W., van Oyen, H. G., van DE Poll, N. E., \& Boer, G. J. (1983). Failure of dexamethasone to influence sex differences in acquisition of discriminated lever press avoidance. Pharmacology, Biochemistry \& Behavior, 19, 599-604.

Hennessy, J. W., Smotherman, W. P., \& Levine, S. (1980). Investigations into the nature of dexamethasone and ACTH effects upon learned taste aversion. Physiology \& Behavior, 24, 645-649.

LeMagnen, J., Marfaing-Jallat, P., Miceli, D., \& Devos, M. (1980). Pain modulating and reward systems: A single brain mechanism? Pharmacology, Biochemistry \& Behavior, 12, 729-733.

Levine, S., \& Levin, R. (1970). Pituitary-adrenal influences on passive avoidance in two inbred strains of mice. Hormones \& Behavior, 1, 105-110.

LowY, M. T., \& YIM, G. K. W. (1982). Drinking, but not feeding is opiate sensitive in hamsters. Life Sciences, 30, 399-401.

Maren, S., Patel, K., Thompson, R. F., \& Mitchell, D. (1993). Individual differences in emergence neophobia predict magnitude of perforant-path long-term potentiation (LTP) and plasma corticosterone levels in rats. Psychobiology, 21, 2-10.
Mitchell, D., Fairbanks, M., \& Laycock, J. D. (1977). Supression of neophobia by chlorpromazine in wild rats. Behavioral Biology, 19, 309-323.

Mitchell, D., Kirschbaum, E. H., \& Perry, R. L. (1975). Effects of neophobia and habituation on the poison-induced avoidance of exteroceptive stimuli in the rat. Journal of Experimental Psychology: Animal Behavior Processes, 104, 47-55.

Mitchell, D., Koleszar, A. S., \& Scopatz, R. A. (1984). Arousal and T-maze choice behavior in mice: A convergent paradigm for neophobia constructs and optimal arousal theory. Learning \& Motivation, 15, 287-301.

NachmanN, M., Rauschenberger, J., \& Ashe, J. H. (1977). Stimulus characteristics in food aversion learning. In N. W. Milgram, L. Krames, \& T. M. Alloway (Eds.), Food aversion learning (pp. 105-131). New York: Plenum

PraTT, N. C., \& LisK, R. D. (1990). Dexamethasone can prevent stressrelated litter deficits in the golden hamster. Behavioral \& Neural Biology, 54, 1-12.

ROCHFORD, J. (1992). The effects of clonidine and yohimbine on noveltyinduced hypoalgesia. Psychobiology, 20, 163-165.

ROCHFORD, J., \& STEWART, J. (1987). Activation and expression of endogenous pain control mechanisms in rats given repeated nociceptive tests under the influence of naloxone. Behavioral Neuroscience, 101, 87-103.

RoziN, P. (1977). The significance of learning mechanisms in food selection: Some biology, psychology, and sociology of science. In L. M. Barker \& M. Domjan (Eds.), Learning mechanisms in food selection (pp. 557-589). Waco, TX: Baylor University Press.

Russell, P. A. (1973). Relationships between exploratory behavior and fear: A review. British Journal of Psychology, 64, 417-433.

ShERMAN, J. E. (1979). The effects of conditioning and novelty on the rat's analgesic and pyretic response to morphine. Learning \& Motivation, 10, 381-418.

WoNG, R. (1985). Saccharin preference of hamsters, gerbils and rats in the "two-food" situation. Behavioural Processes, 10, 87-99.

Wong, R., \& MCBRIDE, C. B. (1993). Flavor neophobia in gerbils (Meriones unguiculatus) and hamsters (Mesocricetus auratus). Quarterly Journal of Experimental Psychology, 46B, 129-143.

Woods, S. C. (1991). The eating paradox: How we tolerate food. Psychological Review, 98, 488-505.

(Manuscript received November 18, 1992; revision accepted for publication January 14, 1994.) 